\section{Erkundigen Sie sich immer nach dem Schlaf!}

$\mathrm{K}$ inderärzte sollten bei ihren Patienten grundsätzlich an Schlafprobleme denken. Die betroffenen Kinder zeigen nicht nur Symptome wie Müdigkeit, sondern auch unspezifische Symptome wie schlechte Schulleistungen und Konzentrationsstörungen, hinter denen ein gestörter Schlaf stecken kann. „Das Wichtigste ist es, den Schlaf überhaupt erst einmal in die kinderärztliche Anamnese mit aufzunehmen“, sagte Prof. Dr. Uwe Mellies vom Universitätsklinikum Essen. Liegen Beschwerden vor, werden diese von den Familien häufig nicht bewusst wahrgenommen. Deshalb sollten Kinderärzte immer gezielt nachfragen: Wann geht das Kind ins Bett? Wann schläft es ein? Ist der Schlaf erholsam?

Die Diagnostik von Kindern mit Schlafstörungen beinhaltet eine strukturierte Anamnese, eine körperliche Un- tersuchung und es wird nach Grunderkrankungen geforscht, die hinter einer Insomnie stecken könnten. Häufige Ursachen sind körperliche Erkrankungen wie nächtliches Husten, Asthma bronchiale, allergische Rhinokonjunktivitis oder auch Schmerzen. Medikamente wie zum Beispiel $\beta$-2-Mimetika können ebenfalls ein Grund sein. Außerdem muss auf Adenoide und Tonsillen geachtet werden.

Protokolle oder Schlaftagebücher können bei der Diagnostik sehr hilfreich sein. Diese sind auf der Homepage der Deutschen Gesellschaft für Schlafforschung und Schlafmedizin (www.dgsm. de) erhältlich. Die Eltern können zusätzlich aufgefordert werden, den Schlaf ihres Kindes zu beobachten. „Dies ist eine einfache Maßnahme“, erklärte Mellies. Idealerweise in der zweiten Nachthälfte, sollte nach dem Schlaf der Kinder ge-

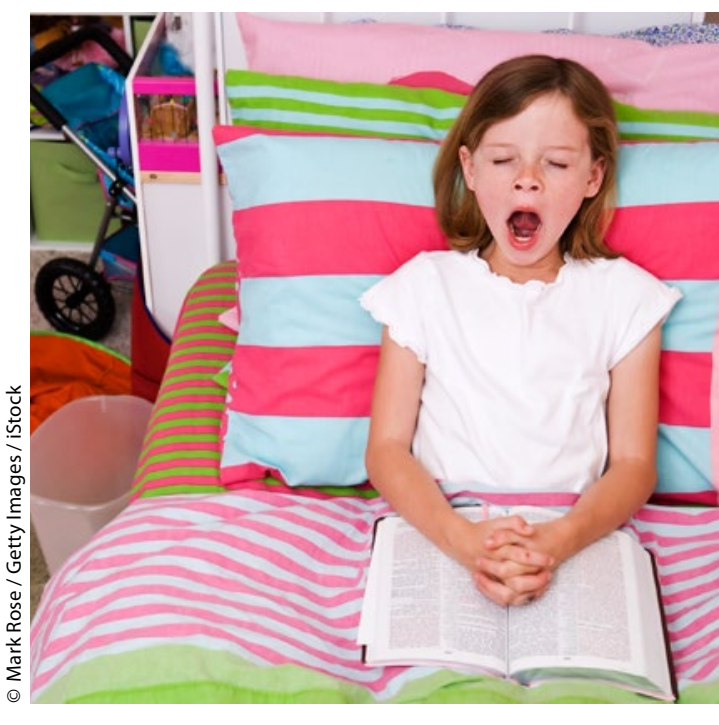

schaut werden. „Heutzutage kann dies wunderbar dokumentiert werden, da fast jeder ein Smartphone hat.“ Die Eltern sollten das Video dann beim nächsten Arztbesuch mitbringen.

\title{
Drei Seltene im Überblick
}

$\mathrm{R}$ und 6.000-8.000 seltene Erkrankungen sind bereits identifiziert. Jede Woche kommen circa fünf neue hinzu. Drei nicht ganz so selten vorkommende Orphan Diseases sind Morbus Fabry, Morbus Gaucher und Morbus Hunter (Übersicht im Kasten).

Die ersten Anzeichen für einen Morbus Fabry können bereits im 2. Lebensjahr, jedoch auch erst im Erwachsenenalter auftreten. Typische frühe Symptome sind brennende Schmerzen, Wärmeintoleranz, Hautläsionen (Angiokeratome) und gastrointestinale Beschwerden. Mit zunehmendem Alter werden die Symptome schwerwiegender und es kann zu Nieren-, Herz- und zerebrovaskulären Problemen kommen. Die lysosomale Speichererkrankung wird durch eine oder mehrere Mutationen im GLA-Gen hervorgerufen, das für $\alpha$-Galactosidase A kodiert. Die Lebenserwartung dieser progressiven Multisystemerkrankung ist bei Männern gewöhnlich um 20 Jahre, bei Frauen um 15 Jahre verkürzt.

Eine weitere lysosomale Speichererkrankung ist der Morbus Gaucher. Er wird autosomal-rezessiv vererbt. Dabei ist die Aktivität des Enzyms $\beta$-Glukocerebrosidase vermindert, sodass es zu einer Glukosylzeramid-Akkumulation in den Makrophagen kommt. Es können mehrere Organsysteme befallen sein: Splenomegalie, Hepatomegalie, Beeinträchtigung der Knochen (Knocheninfarkte), Anämie, Thrombopenie sind mögliche Zeichen, aber auch Lunge oder Gehirn können beteiligt sein.

Der Morbus Hunter ist eine Blickdiagnose. Physische Symptome bei den betroffenen Kindern sind vergröberte $\mathrm{Ge}$ -

\section{Kurzcharakteristik}

Morbus Fabry: Extremitätenschmerzen, Gelenkschmerzen, Kleinwuchs

Diagnosesicherung: erniedrige a-Galaktosidase-Aktivität, molekulargenetische Diagnostik

Morbus Gaucher: Extremitätenschmerzen, Nasenbluten, Hepatosplenomegalie Diagnosesicherung: erniedrigte $\beta$-Glukozerebrosidase-Aktivität, erhöhte sichtszüge und eine vergrößerten Zunge. Häufig kommt es zu einem Makrozephalus, Hepatosplenomegalie und Umbilikal- und Inguinalhernien. Kleinwuchs, Gelenkkontrakturen und knöcherne Deformitäten sind ebenfalls häufig sowie wiederholte Otitiden, chronisches Nasenlaufen und geschwollene Mandeln und Polypen. Auf der Haut können „pebbly papules“ (blasse, meist gruppiert stehende Papeln) auftreten, die sich spontan zurückbilden können.

$n z$

Hennermann J, Muschol N. SOKO Diagnose Entdecken und finden Sie seltene Erkrankungen 